\title{
THE PERFORMANCE OF NEURAL NETWORKS IN ASTRONOMICAL IMAGE CLASSIFICATION
}

\author{
S.C. ODEWAHN \\ Department of Astronomy \\ University of Minnesota \\ Minneapolis, MN 55455 \\ U.S.A.
}

\begin{abstract}
The use of neural network pattern recognition techniques in the field of astronomy is reviewed. In assessing the quality of image recognition derived from this method particular attention is given to the problem of star/galaxy discrimination in large digital sky surveys. A two color survey of 9 fields of the first epoch Palomar Sky Survey, centered on the North Galactic Pole, has been performed with the Minnesota Automated Plate Scanner. A set of neural network image classifiers are used to automatically perform star/galaxy discrimination. We assess the efficiency of image classification and sample completeness through comparisons with a variety of independent studies of the NGP area.
\end{abstract}

\section{Introduction}

The APS group has just completed scanning the glass copies of the blue and red ( $O$ and $E$ ) plates above a galactic latitude of $b=20^{\circ}$ in the Palomar Observatory-National Geographic Sky Survey (POSS I). All of the detected images are being cataloged with positions, isophotal shapes, isophotal diameters, magnitudes, and colors. The stellar and non-stellar objects are separated using a set of neural network image classifiers (Odewahn et al. 1992a). The neural network technique is particularly suited to this application. From each detected object we derive a host of quantitative parameters describing the luminosity distribution in the image. Although some of these quantities are clearly superior to others in discerning the type of image in question, a large number of parameters contain some information. As will be described shortly, a neural network allows one to properly weight a large number of parameters in such a way as to extract a best guess for the classification of the image, i.e. whether or not it is stellar or non-stellar. This work demonstrates that a series of well-trained neural network image classifiers may be used to process properly normalized image parameters from multiple sets of POSS fields. The analysis of our present north galactic pole (NGP) survey will serve to demonstrate the reliability of the image classification and survey completeness for the remainder of the APS POSS catalog.

A number of groups have used a variety of techniques to address the general problem of star/galaxy separation as well as the more complex problem of automatic galaxy morphology classification. Spiekermann (1992) used a fuzzy logic classifier to assign morphological types to galaxy images as faint as $J=18$ digitized from IIIa-J Schmidt plates. Fayadd et al. (1992) have used the decision tree technique to perform star/galaxy separation for the POSS II digitization project. The neural network technique used at Minnesota, as applied to star/galaxy 
separation, is described in Odewahn et al. (1992a, 1992b). The use of this technique for active instrument control has been discussed by Angel et al. (1990) and Ozard \& Morbey (1993). In addition, Storrie-Lombardi et al. (1992) have used a neural network to classify galaxy morphological types on the basis of global photometric parameters.

A thorough description of the neural network pattern classification technique is provided in McClelland \& Rumelhardt (1988). Briefly, neural networks are systems of weight vectors, whose component values are established through various machine learning algorithms, which take as input a linear set of pattern inputs. The network output pattern consists of a series of on/off nodes whose configuration determines the classification. Backpropagation networks are supervised learning techniques. The networks start from a random initial state. A set of training patterns is used to 'teach' the network to perform the desired classification function. The training set must contain a representative sample of patterns for each class. Starting from a random initial configuration, the network is used to classify each pattern in the training set. Each time a pattern in the training set is misclassified, an error term is computed and used to adjust the network's configuration. The patterns in the training set are repeatedly presented to the classifier in this manner until the entire training set is correctly classified or until the network is unable to learn any more patterns. For the remainder of this paper, we shall consider the application of a neural network classifier to the problem of star/galaxy separation on multiple Schmidt plate fields.

\section{The Method}

A complete description of the APS scanning procedure is given in Pennington et al. (1993). Following digitization of all images detected above a preset threshold level (generally corresponding to a surface brightness of $24.5 \mathrm{mag} \operatorname{arcsec}^{2}$ in the B-band), a series of postprocessing procedures is used to produce the final catalog of classified objects. The cataloged image parameters, discussed in previously cited papers, are used in various combinations as inputs to a set of neural network pattern classifiers.

Before the acquired sets of matched image parameters may be used for object classification, we must account for systematic plate-to-plate variations. For each POSS I field, we compute the mean value of each classifier parameter in a series of 9-micron-wide diameter bins. Most of these mean curves follow closely the mean relation for the entire system. However, there are occasionally cases where the mean trend for a particular plate departs significantly from the mean trend for the full 9 plate system. Clearly, before any single image classification system may be applied to such a multiple plate system, these variations must be removed by transforming all of the pertinent parameters to a common system. A parameter space normalization technique has been developed to ensure that all image classification parameters are transformed to a mean system before object classification occurs. Normalization factors for each diameter interval are computed using the diameter-binned mean relation for each parameter and the corresponding mean relation derived from the 9 NGP fields. In practice, this normalization technique is applied independently to three radial zones on each plate. By reducing each plate zone independently we greatly reduce the effects of vignetting and radial optical distortion and thereby produce a more consistent catalog of image classifications for the entire field. In Fig. 1 we illustrate the usefulness of this normalization technique. In each case the solid curve represents the expected mean stellar locus in the $\left[D, T_{\text {av }}\right]$ plane defined by the 9 plate system. As can be seen in the raw data of Fig. 1, the observed stellar locus clearly lies well off the mean relation. Following 

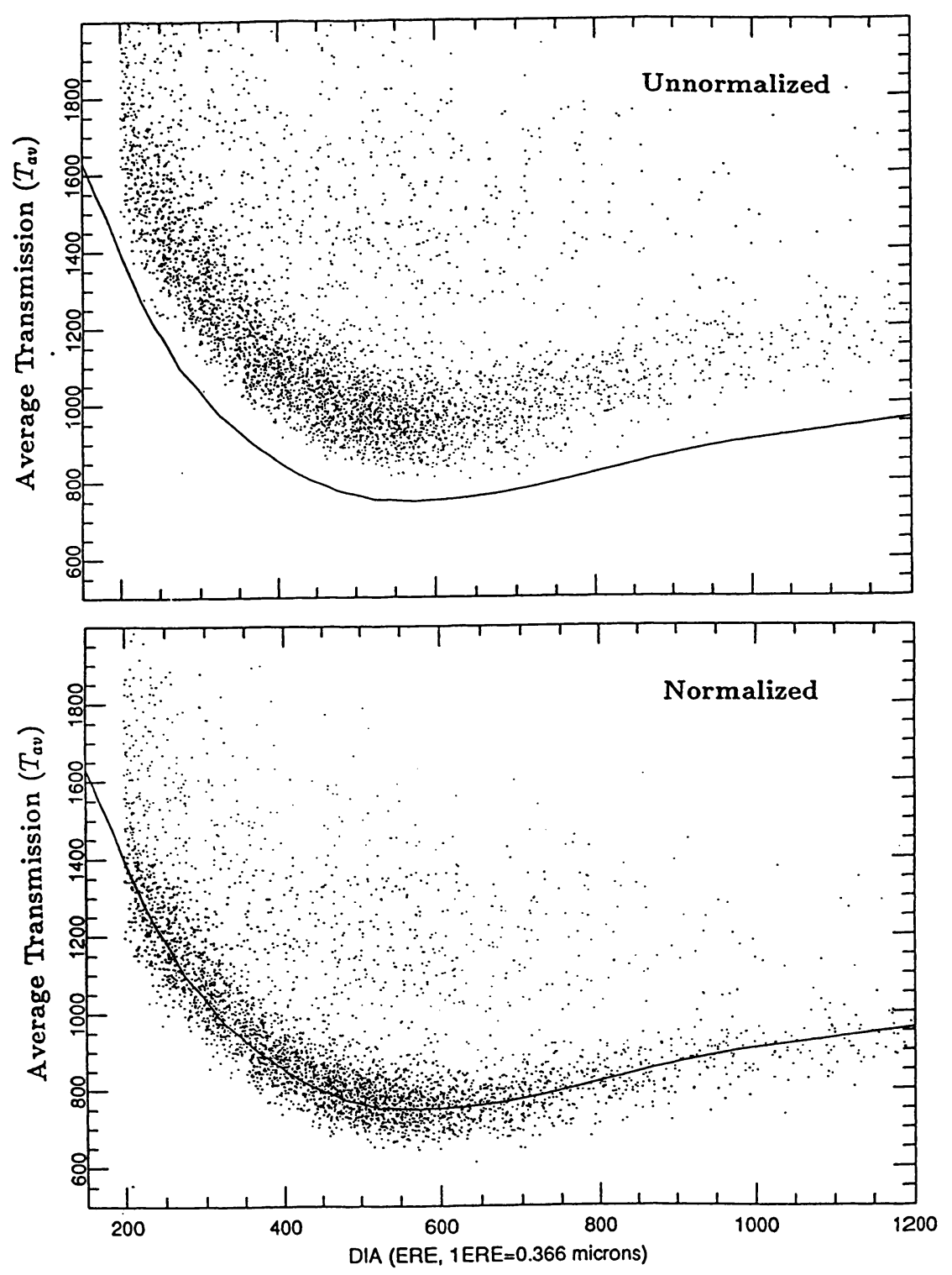

Figure 1. The raw $\left[T_{e v,}, D\right]$ plane for a sample of images from the POSS field P380 is shown in the top plot. The solid line represents the expected ridge-line of the stellar locus for the adopted mean system. The unnormalized P380 data clearly do not follow this trend. For the normalized data of the lower plot, the high density region of points which is the stellar locus is in good agreement with the solid line representing the expected ridge-line of the stellar locus. 
normalization, the observed stellar locus is now centered on the mean relation.

For the present work, we are using a set of three network classifiers to process images in three diameter regimes. An iterative training process known as backpropagation is used to establish the weight values in each network node. This technique requires a sample of 'training' patterns with known object classifications. In a single training pass the network is presented with the entire training sample. In all cases we use two hidden node layers. The primary source of our training data was the star/galaxy sample taken from P323 and described in Odewahn et al. (1992a). Future work will incorporate samples drawn from other POSS plates to train more robust networks. Unlike past networks, we have used smaller input vectors consisting of the most effective image parameters for object discrimination. As discussed by Salzberg (1992), the inclusion of parameters in the input vector which do not convey useful information about the object class serves only to add noise to the output vector and hence the predicted classifications.

\section{Quality of the Image Classification}

In order to assess the reliability of the neural network image classifications, it is necessary to obtain an independent sample of image classifications for the purpose of comparison. For this purpose, we have used the following sources of data:

1) direct visual classification using POSS glass copy plates;

2) FOCAS analysis of CCD images scattered throughout the NGP survey area;

3) the deep Mayall 4-meter photographic survey of Selected Area 57 by Koo et al. (1986); and

4) the CCD drift scan survey of Kent et al. (1993).

In all four cases, we compare the classes between objects in common with the APS neural network sample by computing the percentage agreement ( $S=$ (Number Agreeing)/(Total Number)) between classification sets. For the relatively large comparison samples presented here, it is possible to subdivide the data into different diameter ranges and study the degradation of the automatic classification system with decreasing image signal and resolution. The results of these systematic studies, the success rate function $S(D)$, will be used to assess the amount of catalog contamination due to improper classification at the faint end of the survey.

We have compared independent sets of visually derived star/galaxy catalogs for the nine POSS I fields discussed in this work and have determined that these samples are reliable at the $90 \%$ level down to an approximate magnitude of $O=19$. As a first check, we use these catalogs to test the APS neural network classifications. In Fig. 2 we plot the percentage agreement, $S$, as function of diameter for the nine NGP fields. These results are based on the results of the $O$ plate classifications only.

A set of E plate image classifiers were trained using data collected from the visually inspected $O$ plate data of the previous section. These images, drawn from the entire NGP survey, were divided into training and testing samples for each of the three diameter regimes used in the $O$ plate classifiers. Using the same image parameters and network architectures as the O-band networks, a set of E-band image classifiers was developed. Hence, each matched image in the survey was classified twice, once for each of two (O and $E)$ plate images. As a second check, we compare the $O$ and $E$ band classification sets for each plate. We find the $O$ and $E$ plate classification sets derived in this manner to be consistent above the $90 \%$ level for the magnitude 


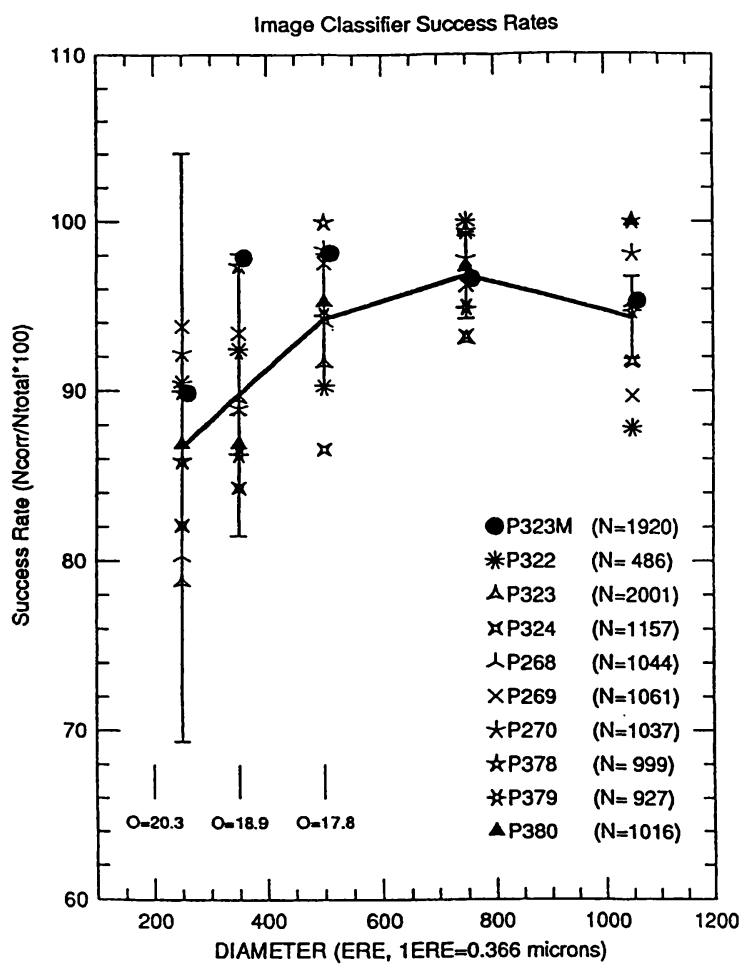

Figure 2. The percentage of agreement, $S$, between visually classified data sets and the neural network classification sets for plates in the APS survey of the NGP region. The set marked as P323M is the mean catalog of visual classifications derived from 4 independent sets of visual classifications from this field. Note the marked improvement of the mean visual set over the other sets which were derived from single classifiers.

range of $20 \leq 0 \leq 16$.

We have analyzed 16 calibrated CCD images centered on 7 Abell clusters in our NGP survey area kindly provided by $\mathrm{N}$. Weir in advance of publication. In the case of 5 clusters both Gunn $\mathrm{g}$ - and r-band images were reduced. Two $\mathrm{g}$-band images each were analyzed for the remaining two clusters. These images were reduced using the Faint Object Classification and Analysis System (FOCAS) described by Jarvis \& Tyson (1981).

In Fig. 3 we compare our CCD (FOCAS) and APS (neural network) derived object classifications. Image classification remains consistent at the $90 \%$ level to approximately $B=19$. In the range $19<B<20$ the percentage agreement drops to a level between $80 \%$ and $90 \%$. It is worth noting that the FOCAS data set comparison represents a somewhat more stringent test for the APS sample. Four of the seven CCD fields are located near the edge of the Schmidt plate field, an area where the quality of the APS image classifications will be degraded by vignetting and other image distortion effects. Restricting our comparison analysis to these 4 fields at the 


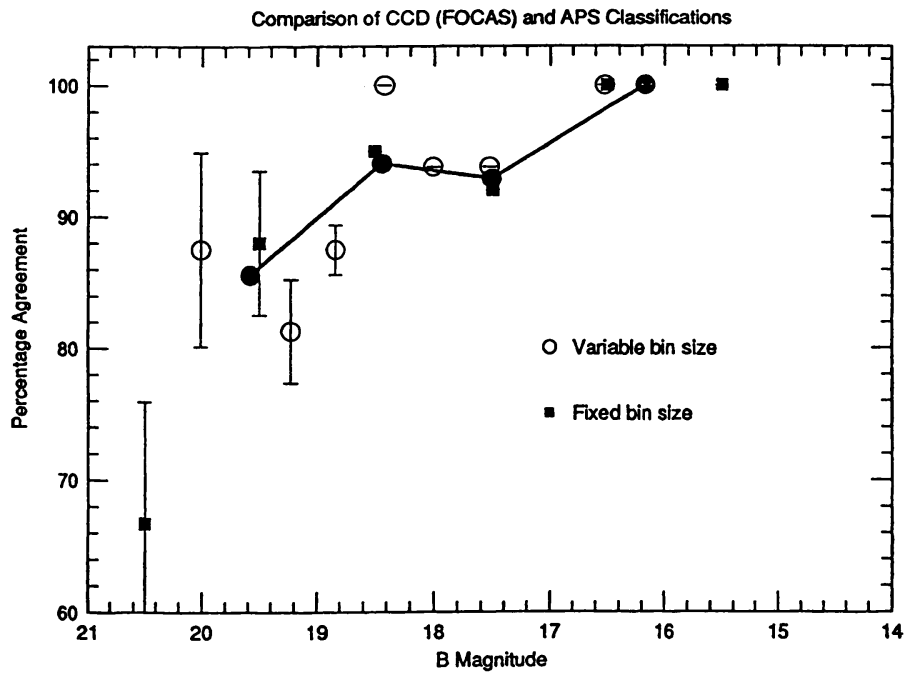

Figure 3. The percentage of agreement between CCD (FOCAS) and APS (neural network) derived object classifications. The open circles represent mean values computed with variable magnitude binning intervals established by collecting sets of 16 objects from a magnitude sorted list. The solid squares represent mean values computed in 1 magnitude wide binning intervals. The large (connected) solid circles represent the mean of the combined distributions. Image classification remains consistent at the $90 \%$ level to approximately $B=19$. In the range $19<B<20$ the percentage agreement drops to a level between $80 \%$ and $90 \%$.

plate edge (A1547, A1551, A1667 and A1746) reduces the number of objects to only 67, but we are able to estimate that for the images fainter than $B=19$ the mean success rate drops to between $75 \%$ and $85 \%$ while for the three fields near the plate center the success rates lie above 90\%. Assuming that the combined APS and FOCAS classification errors add in quadrature, we may conclude that the APS classifications are reliable to the $90 \%$ level down to $B=20$ for the central $2^{\circ}$ radial field.

Past studies of the NGP area include a deep photographic survey of Selected Area 57 by Koo et al. (1986) (hereafter referred to as the SA57 sample) and a 12' wide drift scan survey centered on $\delta=29.5^{\circ}$ by Kent (1993). These surveys were used as independent sources of object classifications for the purpose of confirming the validity of the APS neural network estimates. The neural network classifications derived with the APS agree to better than the $90 \%$ level with the SA57 sample down to a range of $20 \leq 0 \leq 19.5$. Similarly encouraging results were obtained in a comparison with the CCD strip survey of Kent et al. (1993), which contained galaxy identifications for objects on the 3 central plates of the NGP survey. 


\section{Conclusion}

We have conducted a two-color survey of 9 POSS fields centered on the NGP using the Minnesota Automated Plate Scanner. Comparing our APS survey with a number of independent sources of object classifications spread over the NGP area, we have shown that the APS neural network classification system effectively separates stars and galaxies at the level of a $90 \%$ success rate down to the range $19.5<B<20.0$. It is important to note that through a simple normalization of several key image parameter spaces for each photographic plate, a single set of well trained neural network image classifiers, working in three image diameter regimes, has been used to classify objects surveyed on 9 different Schmidt plates covering 324 square degrees. By directly comparing the APS classifications with those derived by visual estimates, FOCASreduced CCD surveys, and a deep 4-meter photographic survey, we find no plate-to-plate variation in the effectiveness of our star/galaxy discrimination.

\section{References}

Angel, R., Wizinowich, P., Lloyd-Hart, M. and Sandler, D., 1990. Nature, 348, 221.

Fayyad, U., Doyle, R., Weir, N. and Djorgovski, S., 1992. Caltech preprint.

Jarvis, J.F. and Tyson, J.A., 1981. Astron. J., 86, 476.

Koo, D.C., Kron, R.G., Nanni, D., Trevese, D. and Vignato, A., 1986. Astrophys. J., 91, 478.

McClelland, J.L. and Rumelhardt, D.E., 1988. 'Explorations in Parallel Distributed Processing', MIT Press, Cambridge, MA.

Odewahn, S.C., Humphreys, R.M., Aldering, G. and Thurmes, P.M., 1992a. Publ. Astron. Soc. Pacific, in press.

Odewahn, S.C., Stockwell, E.B., Pennington, R.M., Humphreys, R.M. and Zumach, W.A., 1992b. Astron. J., 103, 318.

Ozard, S. and Morbey, C., 1993. Publ. Astron. Soc. Pacific, 105, 625.

Pennington, R.L., Humphreys, R.M., Odewahn, S.C., Zumach, W.A. and Thurmes, P.M., 1993. Publ. Astron. Soc. Pacific, 105, 521.

Salzberg, S., 1992. Johns Hopkins University Preprint.

Spiekermann, G., 1992. Astron. J., 103, 2102.

Storrie-Lombardi, M.C., Lahav, O., Sodre, L.J. and Storrie-Lombardi, L.J., 1992. Mon. Not. R. astron. Soc., 258, 8. 